\title{
A Compact UWB Bandpass Filter using Hybrid Fractal Shaped DGS
}

\author{
${ }^{1}$ Babu Lal Shahu \\ ${ }^{1}$ Department of Electronics and Communication Engineering, Birla Institute of \\ Technology, Mesra, Deoghar Campus, Deoghar-814142, India \\ Email: ${ }^{1}$ sahu.babulal@gmail.com \\ ${ }^{2}$ Neela Chattoraj, ${ }^{3}$ Srikanta Pal, ${ }^{4}$ Dileep Kumar Upadhyay \\ ${ }^{2,3,4}$ Department of Electronics and Communication Engineering, Birla Institute of \\ Technology, Mesra Ranchi-835215, India \\ Emails: ${ }^{2}$ nila_chwdhry@yahoo.com, \\ 3pal_srikanta@yahoo.co.uk \\ 4dileep_18@rediffmail.com
}

\begin{abstract}
In this paper, a novel and compact ultra wideband (UWB) bandpass filter (BPF) based on fractal-shaped defected ground structure (DGS) is designed and developed. It is made of stepped impedance lines and a composite right/left handedtransmission line (CRLH-TL) constructed with capacitive gap in conductor strip and a hybrid fractal-shaped ring slot in ground plane. The capacitive gap between conductor strip and fractal shaped DGS play the major role for controlling the lower and upper passband cutoff frequencies. The prototype of proposed filter is fabricated and measured. Good agreement is found between simulated and measured results. The proposed UWB bandpass filter has good passband performance with $10 \mathrm{~dB}$ return-loss bandwidth of 2.2 to $10.6 \mathrm{GHz}$, insertion loss better than $1.1 \mathrm{~dB}$ and fractional bandwidth of $131 \%$. Also, the proposed filter obtained a very wide upper-stopband from $11.4 \mathrm{GHz}$ to beyond $20 \mathrm{GHz}$ with rejection skirt better than $30 \mathrm{~dB}$ at both the lower and upper passband cutoff frequencies. Moreover, UWB BPF also shows the flat group delay performance with variation $\leq \mathbf{0 . 7 5} \mathrm{ns}$ in the entire passband. Thus the proposed filter can be a useful candidate for low profile future wireless communication systems.
\end{abstract}

Index Terms - Composite right/left handed transmission line, Defected ground structure, Hybrid fractal, Ultrawide bandpass filter.

\section{INTRODUCTION}

Rapid growth and interest for the development of Ultra wideband (UWB) system is seen since the authorization by U.S. Federal Communications Commission (FCC) for unlicensed use of UWB frequency band from $3.1 \mathrm{GHz}$ to $10.6 \mathrm{GHz}$ for commercial purposes in 2002 [1]. UWB system nowadays becomes most promising technologies for short range low power indoor wireless communication. As an important component in UWB communication systems, the UWB bandpass filter has been rapidly developed. The great potential of UWB system for developing modern transmission system increased the demand for designing filter with low insertion-loss, higher out-ofband rejection capability and flat group delay characteristics within the desirable band. 
Different methods have been developed to achieve UWB filter response with improved performance. Composite right/left handed transmission line (CRLH TL) technique is proposed for designing of a wide variety of microwave circuit for different applications. An UWB filter is implemented using an unbalanced CRLH TL based on zero degree feeding structure [2], the CRLH TL with capacitive cross coupling is used to design UWB filter [3] with improved selectivity, low insertion-loss and compact size. Multimode resonator (MMR) technique in the form of step impedance resonator (SIR) is implemented in [4] for UWB application. Stub loaded MMR is implemented in $[5,6]$ to improve in-band performance by increasing the number of mode within the passband. However, the UWB filter structures usually have an important drawback of introducing spurious responses in the stopband. So improving out-of-band performance of the UWB filter became a research focus nowadays. Different methods have been adopted such as cascading bandpass and band stop filter [7], by changing resonant mode of MMR [8, 9], electromagnetic band gap (EBG) structures $[10,11]$, using defected ground structure (DGS) $[12,15]$. Besides the planar geometries, fractals are very good solution to improve the performance of UWB bandpass filter in terms of size reduction and increasing the bandwidth due to their two most common properties: self similarity and space filling. A compact size UWB BPF is reported in [16] using CRLH-TL and Koch fractal-shape slot in ground with small variation in group delay, but its insertion-loss was poor $(1.5 \mathrm{~dB})$ and also out-of-band rejection skirts were not sharp enough. To improve the passband and out-of-band rejection performance, a modified UWB filter is reported in [17] with Koch island-shaped stepped impedance line and CRLH-TL synthesized using capacitive gap in conductor strip and Koch fractal shaped slot in ground plane.

In this paper, a compact UWB BPF is presented based on stepped impedance microstrip lines and a composite right-left handed transmission line composed of capacitive gap in conductor strip and etching hybrid fractal-shaped ring slot in ground plane. The proposed UWB BPF is able to achieve the $10 \mathrm{~dB}$ return-loss bandwidth from $2.2 \mathrm{GHz}$ to $10.6 \mathrm{GHz}$ with relative bandwidth of $131 \%$. Also the proposed UWB BPF has deep and sharpened out-of-band rejection skirt with wide stopband performance. The proposed UWB filter is designed and optimized using IE3D commercial full-wave electromagnetic (EM) software simulator on RT/Duroid 5880 substrate of dielectric constant 2.2, thickness $1.57 \mathrm{~mm}$ and loss tangent 0.0009 . The prototype of optimized UWB BPF is fabricated and tested for experimental verification.

\section{DESIGN OF PROPOSED UWB FILTER}

Based on space filling properties of fractal structure, a fractal shaped UWB BPF is designed in microstrip technology. The schematic of proposed UWB BPF structure is shown in Fig 1. The proposed structure is designed using stepped impedance lines and CRLH-TL synthesized by etching fractal-shaped ring slot in the ground plane and series capacitive gap in the conductor strip. UWB BPF consists of six sections of stepped impedance lines (denoted by black) on the both sides of 
proposed symmetric structure with series capacitive gap and a fractal-shaped ring slot (denoted by white) in the ground plane (denoted by grey). The fractal-shaped ring slot in the ground plane is constructed by using the first iteration of combination of Minkowski fractal and Koch fractal. The dimensional schematic of proposed UWB filter is shown in Fig.2. For constructing the fractal shaped ring slot initially a square shaped ring slot of size $l_{1} \times w_{1} \mathrm{~mm}^{2}$ is created in ground plane with slot width of $g_{1}$. Further, the square shaped ring slot is modified as Minkowski like fractal of first iteration with arbitrary iteration factor. In the next stage, Koch fractal of first iteration order of $1 / 3$ iteration factor is made on the each side of Minkowski like fractal ring slot. By adjusting the size $\left(l_{1} \times w_{1}\right)$ and slot width $\left(\mathrm{g}_{1}\right)$ of fractal shaped ring slot, a deep and sharpened out-of-band rejection skirt can be obtained. Adjustment of stepped impedance line parameters (L1, L2, L3, L4, L5, L6 and W1, W2, W3, W4) contribute to improve the pass-band and out-of-band stopband performance.

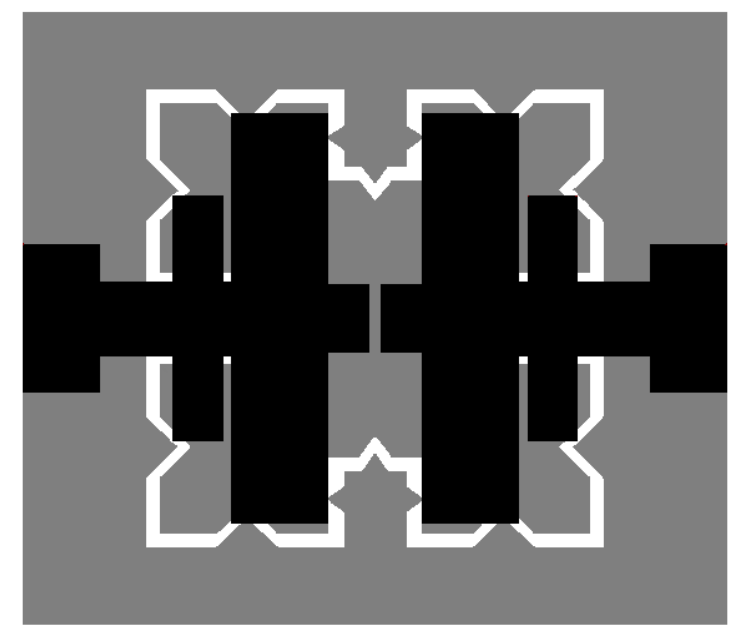

Fig. 1 Layout of proposed UWB bandpass filter

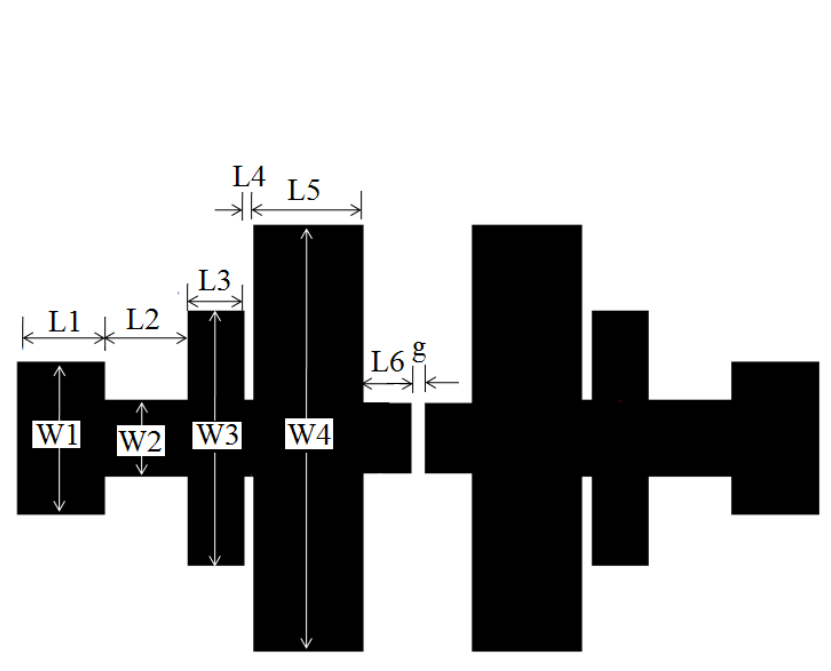

(a) Top view

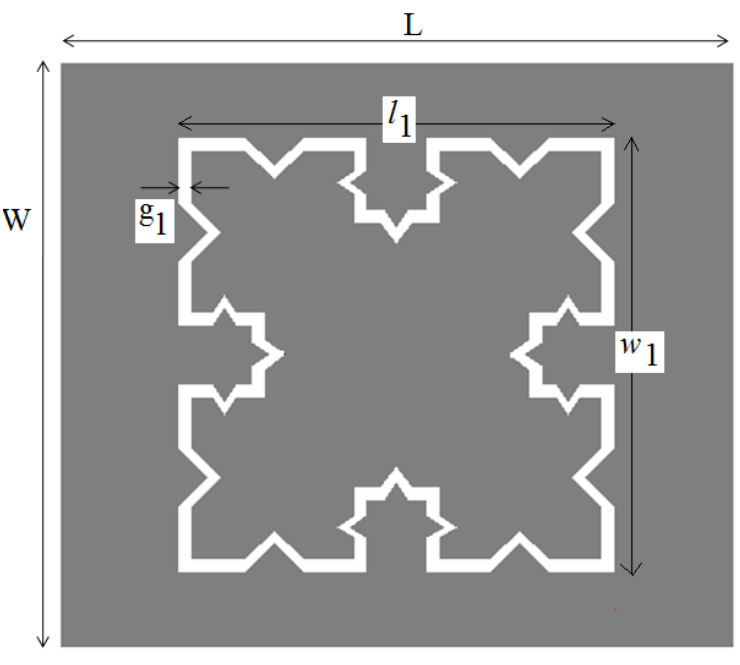

(b) Bottom view

Fig. 2 Dimensional view of proposed UWB bandpass filter, (a) Top view (b) Bottom view 


\section{PARAMETRIC STUDY}

To obtain the best performance and final geometrical parameters the parameters of proposed UWB $\mathrm{BPF}$ are optimized. Parametric study is performed by varying one parameter at a time while keeping the other parameters constant. The parameter W1 is chosen according to $50 \mathrm{ohm}$ impedance calculated using line gauge tool of simulation software IE3D. Simulated S-parameters response with variation in parameter W2 is shown in Fig. 3. It can be seen that the best response is obtained for W2 $=2.4 \mathrm{~mm}$. For values of $\mathrm{W} 2<2.4 \mathrm{~mm}$, the filter is not able to achieve the desired UWB passband performance although the out-of-band stopband performance is good. For value of W2 > 2.4, the insertion-loss in passband is increased with little spurious response in stopband.

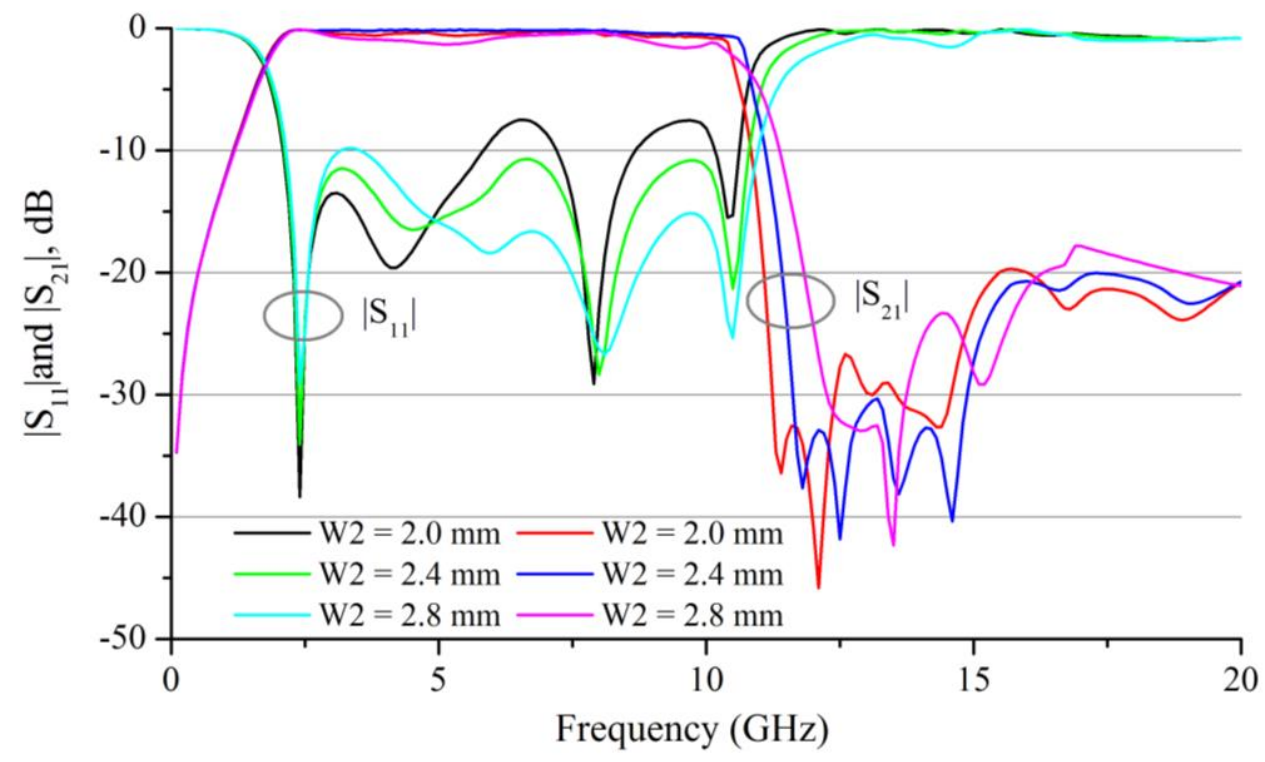

Fig. 3 Simulated return-loss and insertion-loss with variation in parameter W2

The effect of parameter W3 on return-loss and insertion- loss performance is depicted in Fig.4. Best performance is observed for $\mathrm{W} 3=8.0 \mathrm{~mm}$. The passband insertion-loss for $\mathrm{W} 3=7.4 \mathrm{~mm}$ is more and out-of-band spurious response is also observed. For higher value of W3 i.e. for W3 $=8.6 \mathrm{~mm}$, the passband $-10 \mathrm{~dB}$ return-loss bandwidth is decreased although deep and sharp out-of-band rejection skirt is obtained. Similarly for parameter W4, the passband return loss and insertion-loss performance is better for $\mathrm{W} 4=13.4 \mathrm{~mm}$ as presented in Fig.5. In the passband the return-loss is increased at the lower frequency end for $\mathrm{W} 4<13.4 \mathrm{~mm}$ and at the middle of the passband for W4 $>13.4 \mathrm{~mm}$. No much variation in out-of-band performance is observed with variation in parameter W4. 


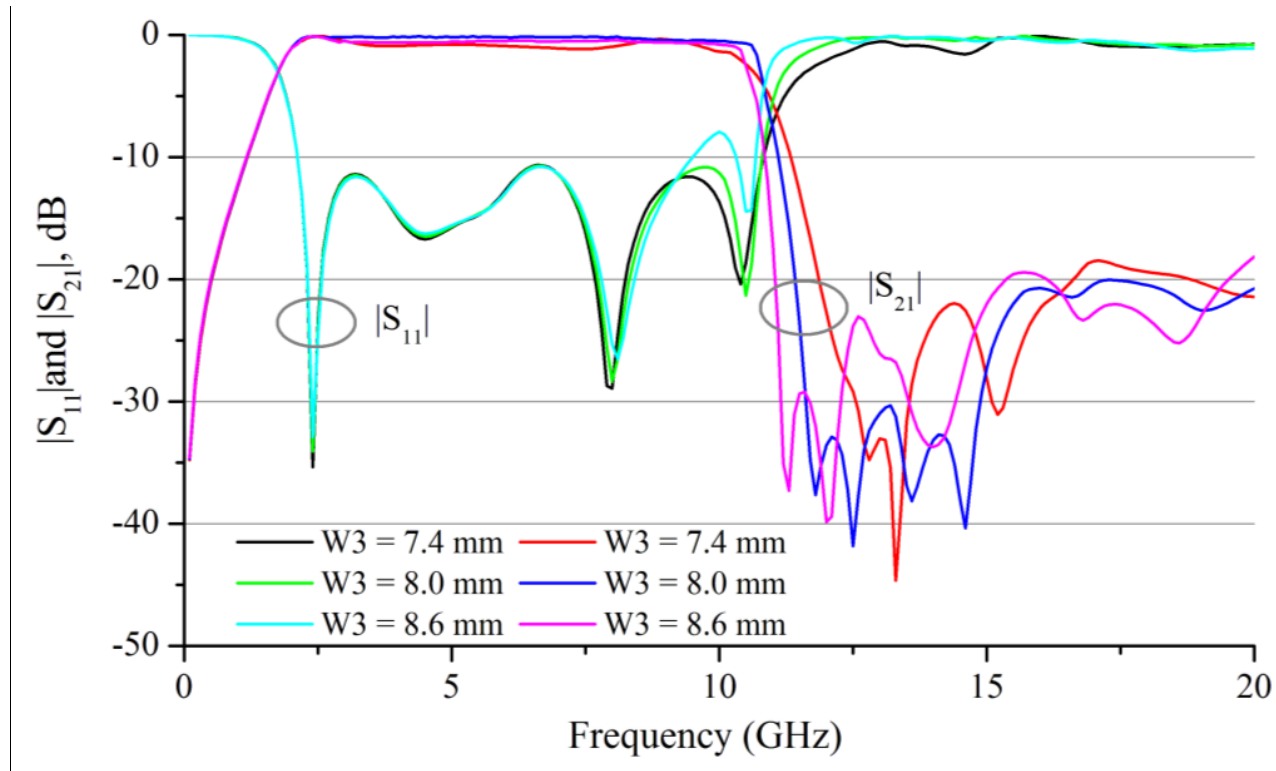

Fig. 4 Simulated return-loss and insertion-loss with variation in parameter W3

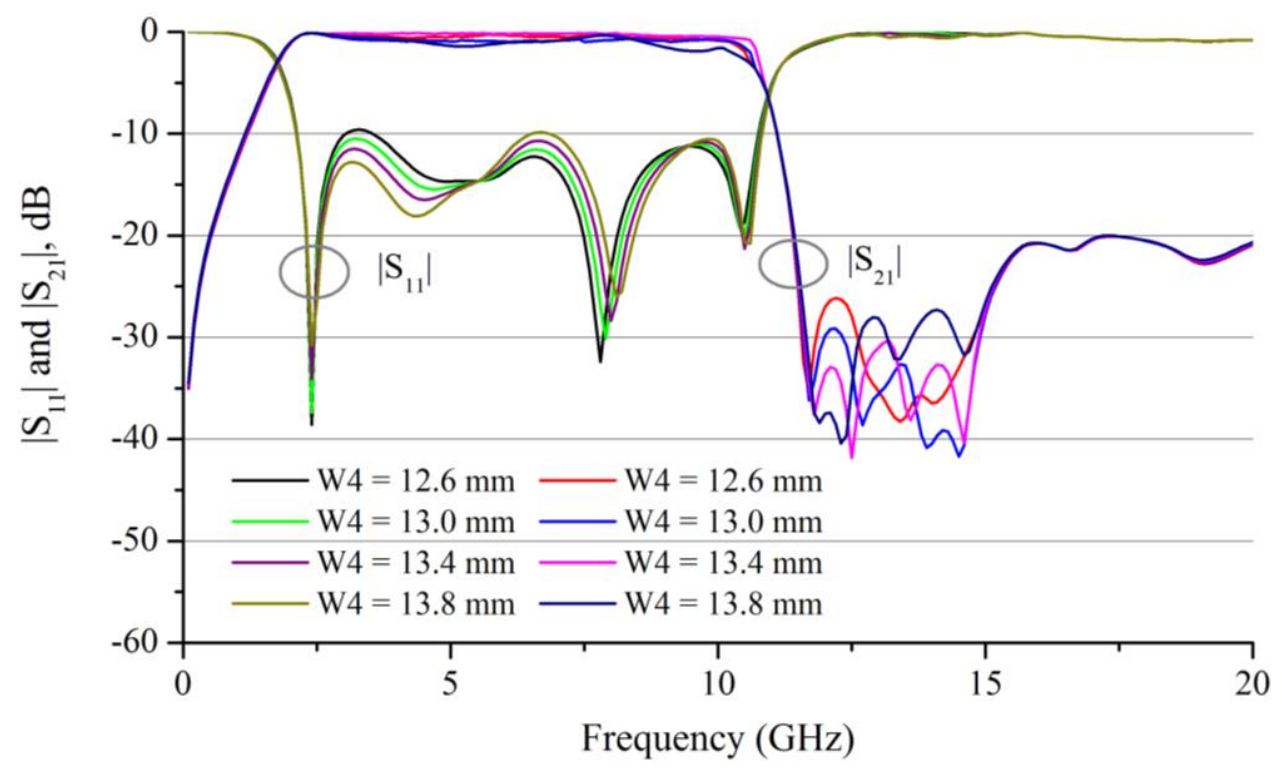

. Fig. 5 Simulated return-loss and insertion-loss with variation in parameter W4

The parameters L1, L2, L3, L4, L5 and L6 are also optimized to improve the performance of proposed UWB filter. Variation in S-parameters with dimensional parameters L1 is shown in Fig. 6. The optimized value of $\mathrm{L} 1$ is taken equal to $2.5 \mathrm{~mm}$ as it gives the best performance in passband as well as in stopband. It is observed that the passband return-loss for $\mathrm{L} 1=3.0 \mathrm{~mm}$ is increased at upper frequency arround at $9.8 \mathrm{GHz}$. For lower value of L1 i.e. at $\mathrm{L} 1=2.0 \mathrm{~mm}$, increase in return-loss is seen in the middle of passband arround at $6.5 \mathrm{GHz}$. Fig. 7 shows the effect of parameter L2 on Sparameters. It is seen that insertion-loss and return-loss performance is better for L2 $=2.4 \mathrm{~mm}$. Lower or higher values of L2 do not fulfill the desired UWB passband performance. Increase in return-loss and insertion-loss is observed for lower or higher value of L2. 


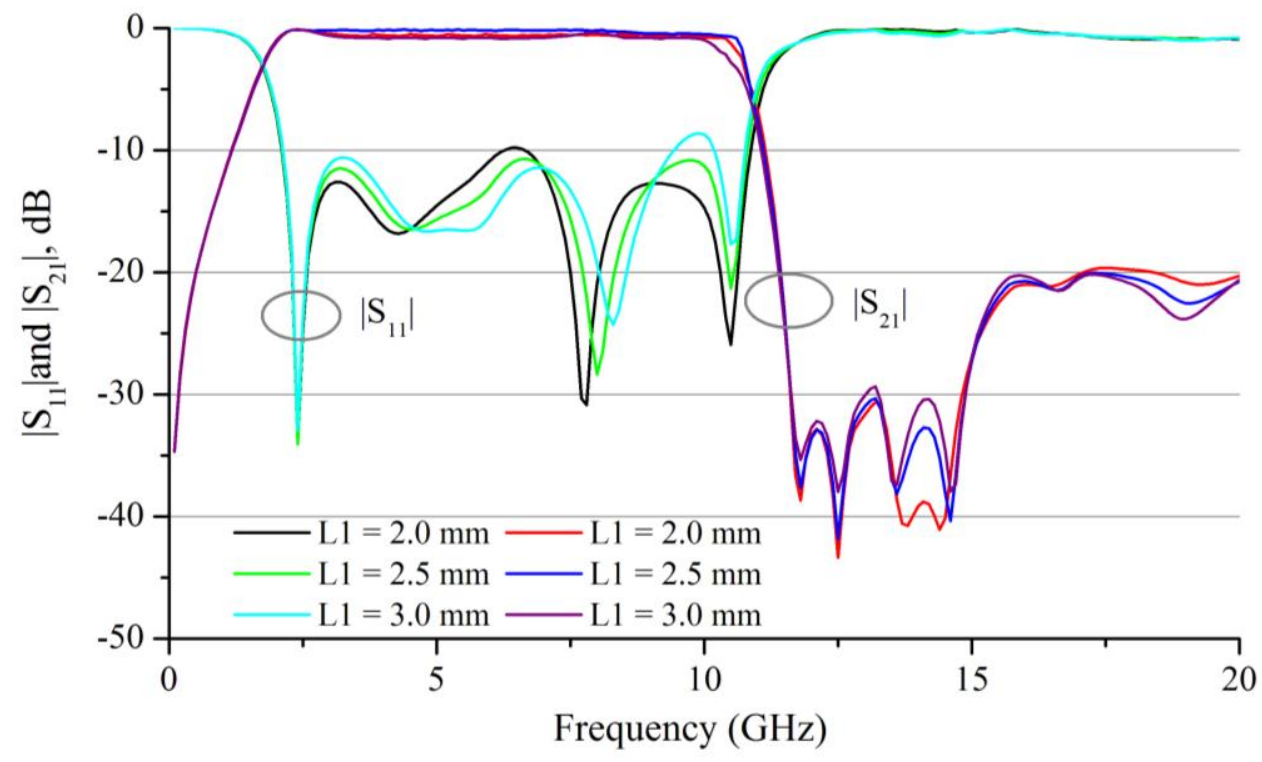

Fig. 6 Simulated return-loss and insertion-loss with variation in parameter L1

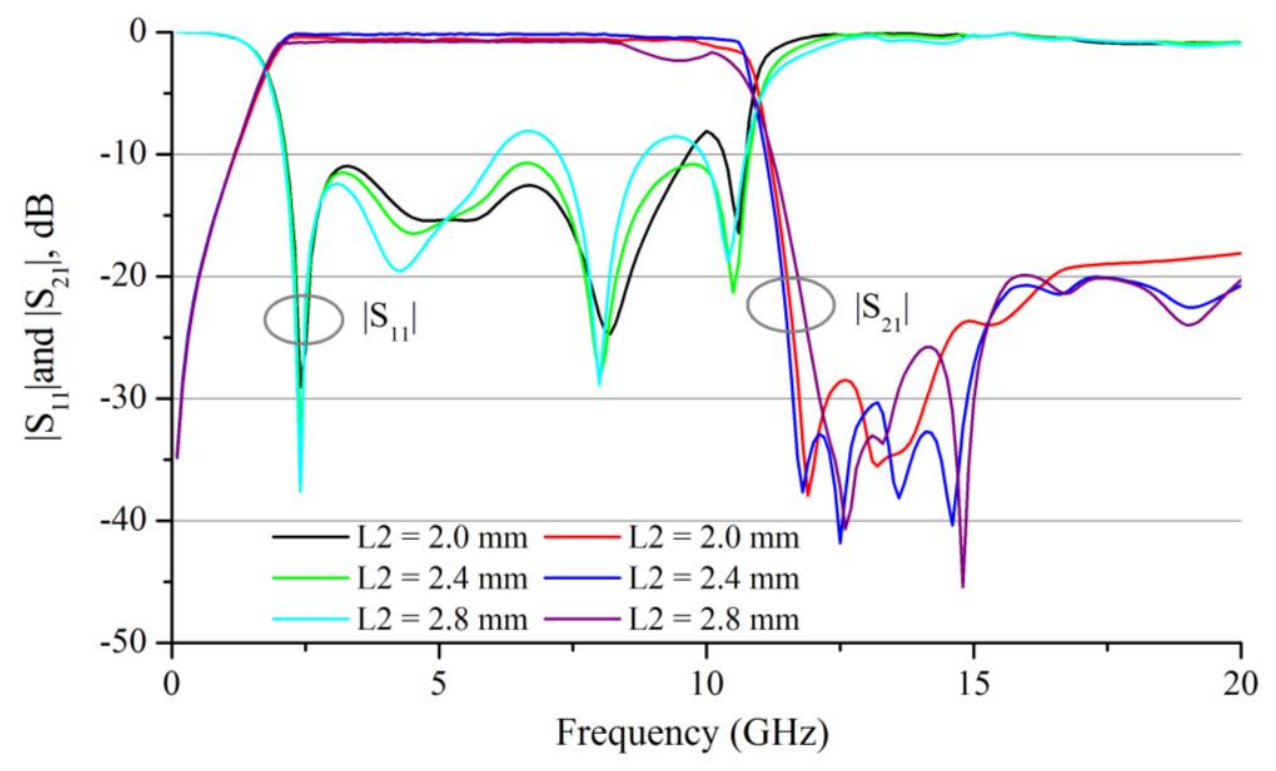

Fig. 7 Simulated return-loss and insertion-loss with variation in parameter L2

Simulated S-parameter performance with variation in parameter L3 is depicted in Fig. 8. Better return-loss and insertion loss characteristics have been observed for $\mathrm{L} 3=1.6 \mathrm{~mm}$. Insertion-loss in the pass band become poor with increased value of L3. Also, the return-loss performance in passband is found to be better for L3=1.6 mm and deteriorate at higher frequency as the value of L3 decreased or increased. In the stopband, slight variation in insertion-loss is found with good return-loss performance. 


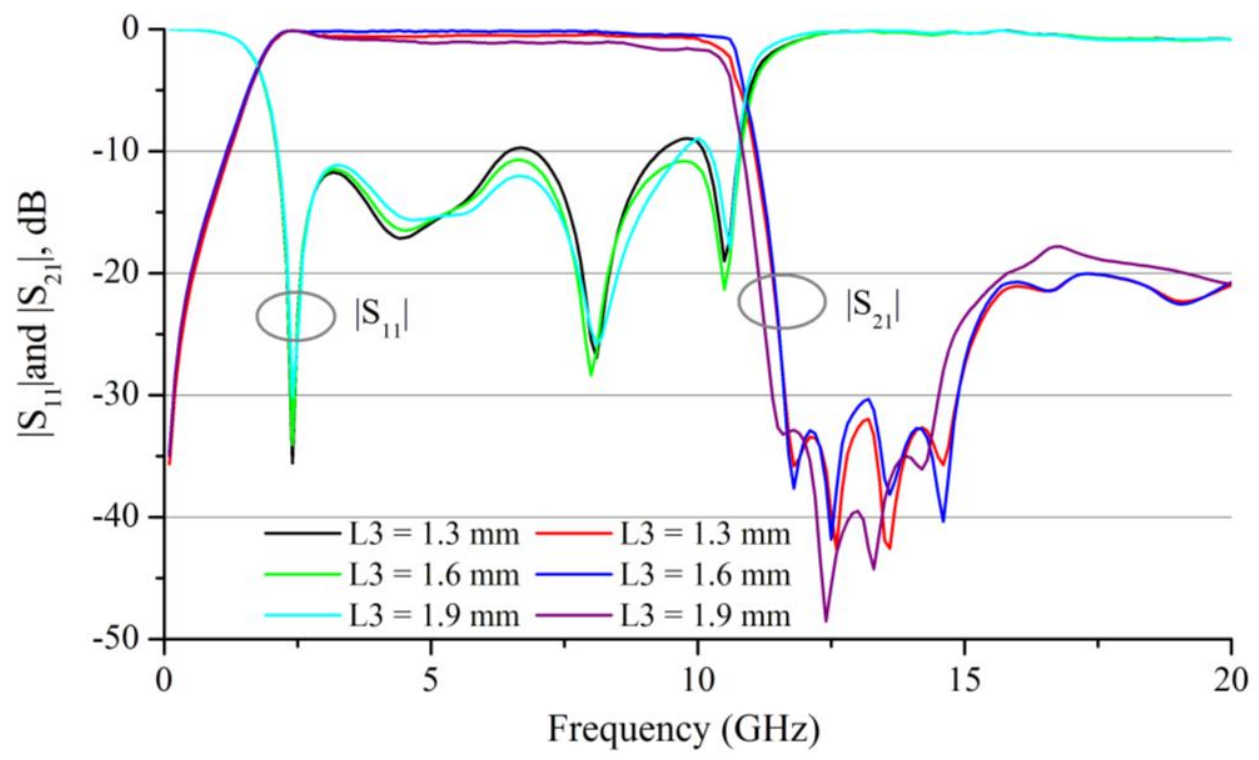

Fig. 8 Simulated return-loss and insertion-loss with variation in parameter L3

Effect of variation of parameter L4 on S-parameter characteristics is plotted in Fig. 9. From Fig. 9, it is seen that the higher value L4 deteriorate the S-parameter performance at higher frequency end in the passband. The optimized dimension of L4 is chosen $0.3 \mathrm{~mm}$ as it provides highest $-10 \mathrm{~dB}$ returnloss bandwidth with sharp out-of-band rejection. The Fig. 10 shows the effect of dimensional parameter L5 on S-parameter characteristics. Better return-loss and insertion-loss response is found for L5 $=3.1 \mathrm{~mm}$. Decrease in $-10 \mathrm{~dB}$ return-loss bandwidth is observed for lower value of L5. For higher value of L5, insertion-loss in the passband is increased. Optimized values of different physical parameters of proposed UWB BPF are summarized in Table I.

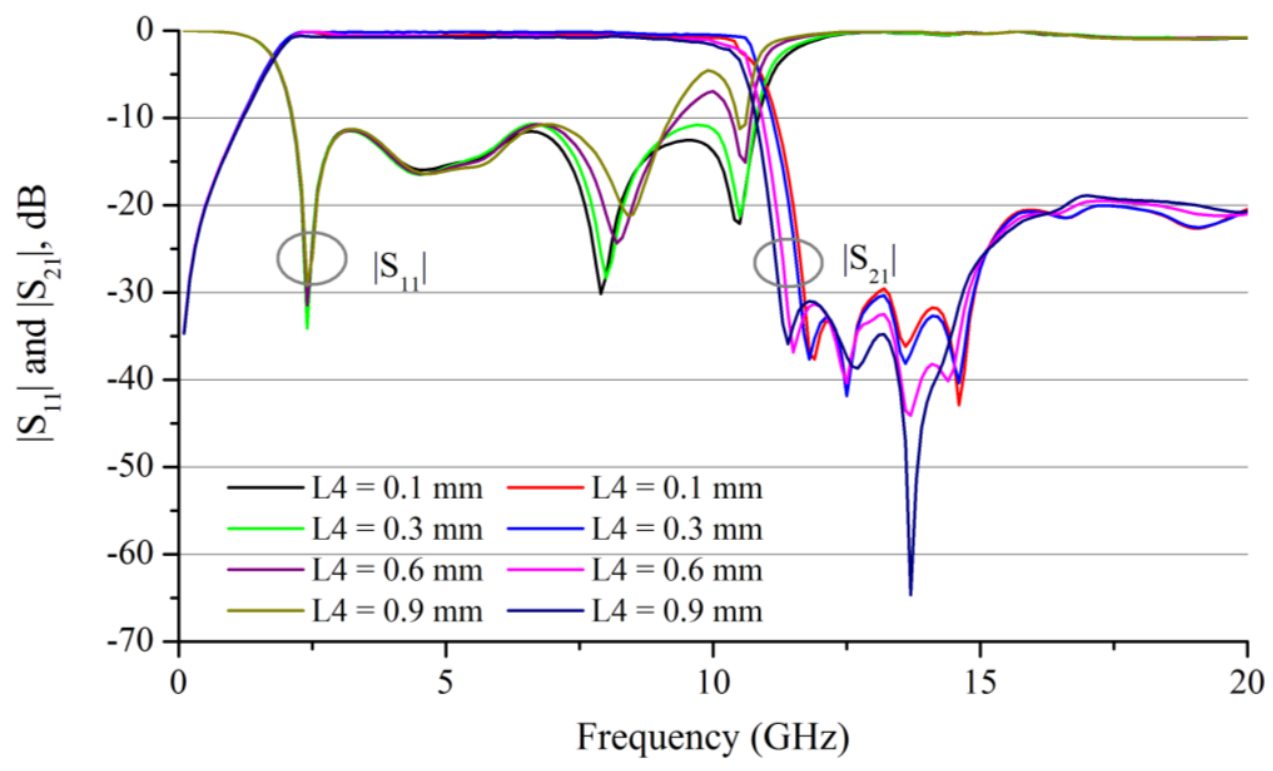

Fig. 9 Simulated return-loss and insertion-loss with variation in parameter L4 


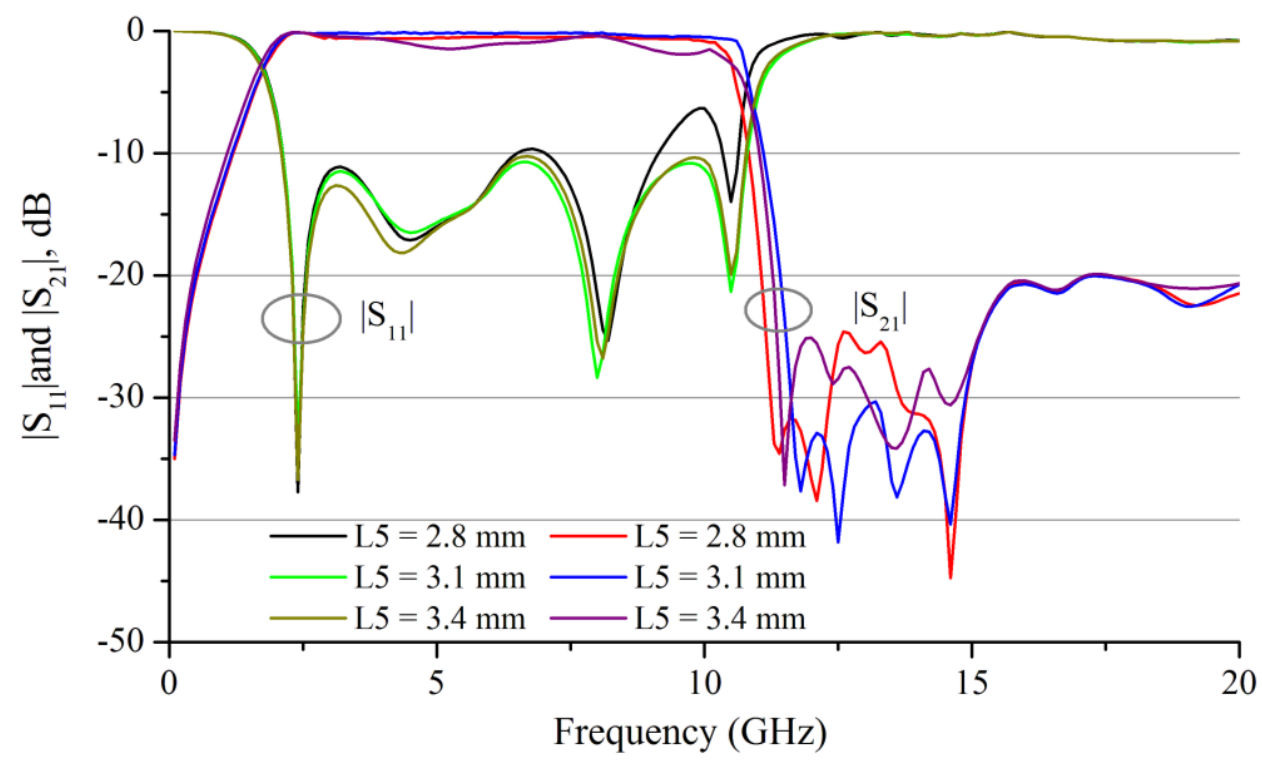

Fig. 10 Simulated return-loss and insertion-loss with variation in parameter L5

TABLE I. OPTIMIZED PHYSICAL PARAMETERS OF UWB BPF SHOWN IN FIG. 2

\begin{tabular}{l|cccccccccccccccc}
\hline Parameter & W1 & W2 & W3 & W4 & L1 & L2 & L3 & L4 & L5 & L6 & g & L & W & $\mathrm{g}_{1}$ & $l_{l}$ & $w_{1}$ \\
\hline Dimension (mm) & 4.8 & 2.4 & 8.0 & 13.4 & 2.5 & 2.4 & 1.6 & 0.3 & 3.1 & 1.4 & 0.4 & 23 & 20 & 0.5 & 15 & 15 \\
\hline
\end{tabular}

Fig. 11 displays the simulated surface current distributions of proposed UWB bandpass filter at three different frequencies $3.1 \mathrm{GHz}, 5.5 \mathrm{GHz}$ and $10.5 \mathrm{GHz}$. It is observed that at $3.1 \mathrm{GHz}$ the current density is mainly concentrated in the ground plane around the fractal-shaped ring slot. At $5.5 \mathrm{GHz}$ the current is concentrated at the top plane in stepped impedance line as well as in ground plane. However at $10.5 \mathrm{GHz}$, more concentration of current is observed in the stepped impedance microstrip line section with little concentration around the ring slot in the ground plane.

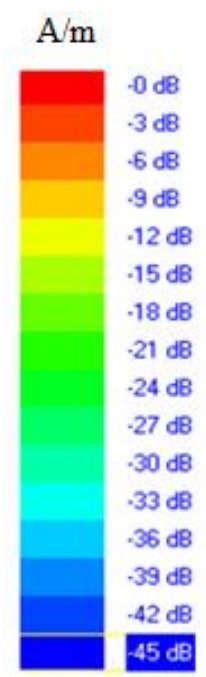

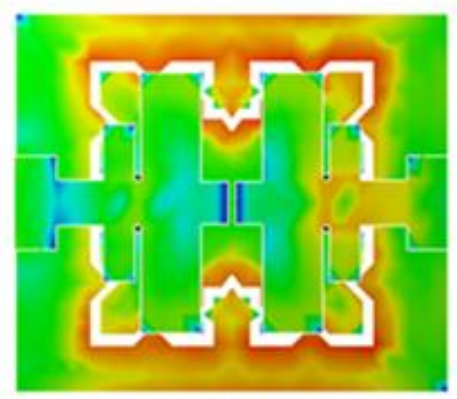

$3.1 \mathrm{GHz}$

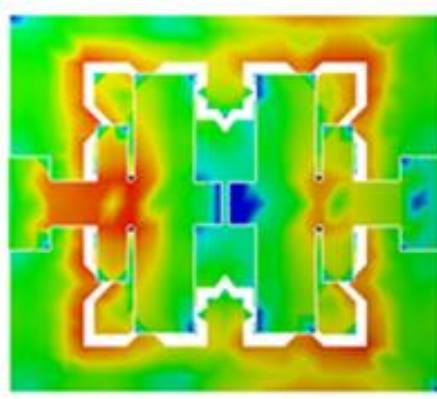

$5.5 \mathrm{GHz}$

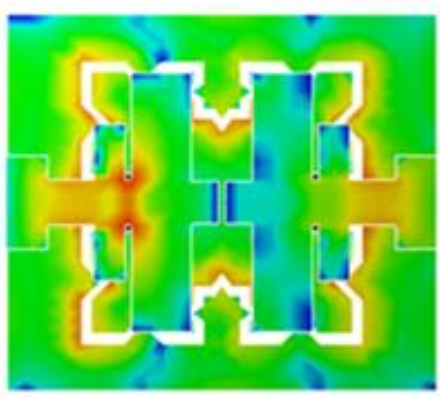

$10.5 \mathrm{GHz}$

Fig. 11 Simulated current distributions 


\section{MEASUREMENT RESULTS AND DISCUSSION}

To validate the performance, a prototype of proposed UWB BPF is fabricated based on optimized dimensional parameters and measured. The photograpgs of top and bottom of fabricated prototype is represented in Fig.12 (a) and (b) respectively. To validate the simulation results the performance parameters of UWB filter are measured and compared with simulated results. The comparison between simulated and measured return-loss and insertion-loss performance is plotted in Fig.13, and are found to be in good agreement with each other. It can be seen that the measured insertion-loss, $\left|\mathrm{S}_{21}\right|$ is better than $1.1 \mathrm{~dB}$ within the entire $-10 \mathrm{~dB}$ return loss passband from $2.2 \mathrm{GHz}$ to $10.6 \mathrm{GHz}$ irrespective of simulated $\left|S_{21}\right|$ which is better than $0.6 \mathrm{~dB}$.

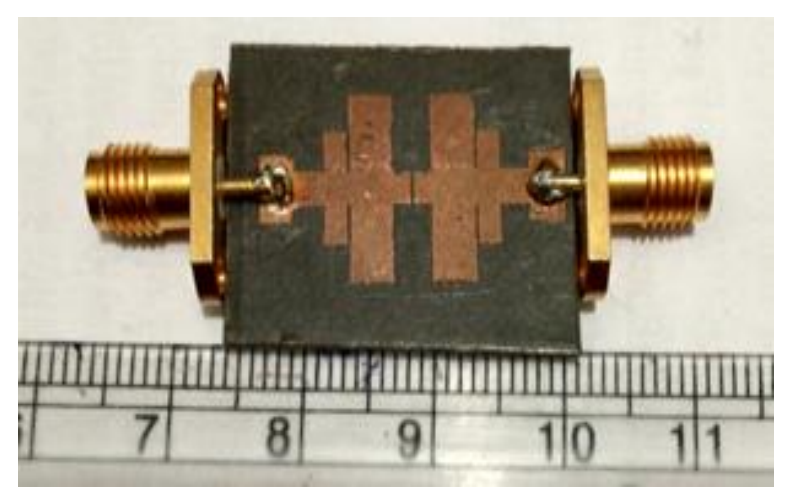

(a) Top view

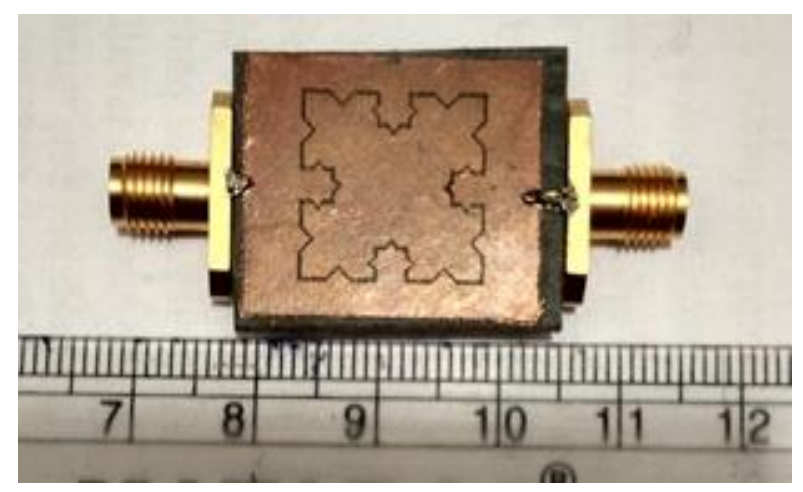

(b) Bottom view

Fig. 12 Fabricated photograph of proposed UWB BPF

Beyond UWB frequency band, deep and sharp skirt is observed with rejection level better than 37 $\mathrm{dB}$ at both lower and upper stopband transition. Also, very wide upper-stopband is obtained from 11.4 GHz to beyond $20 \mathrm{GHz}$. The measured return-loss in upper-stopband is almost flat and agreed with simulated response with performance better than $0.8 \mathrm{~dB}$ extended up to above $20 \mathrm{GHz}$. Also the measured and simulated insertion- loss in this frequency band of interest is found to be better than 20 dB. So the proposed UWB filter has well out-of band rejection performance with very wide upper- 
stopband characteristic. The little discrepancy in simulated and measured results may be due to fabrication tolerances and improper soldering of SMA connector.

The group delay performance for proposed hybrid fractal shaped UWB bandpass filter is plotted in Fig. 14 showing a good agreement between simulated and measured result. It is observed that the measured group delay varies between 1.20 to $1.95 \mathrm{~ns}$ within the desired UWB frequency band while the simulated group delay varies between 1.40 to $1.85 \mathrm{~ns}$. Thus the designed filter is able to achieve maximum group delay variation of $0.75 \mathrm{~ns}$ which shows a good phase linearity and better performance in time domain.

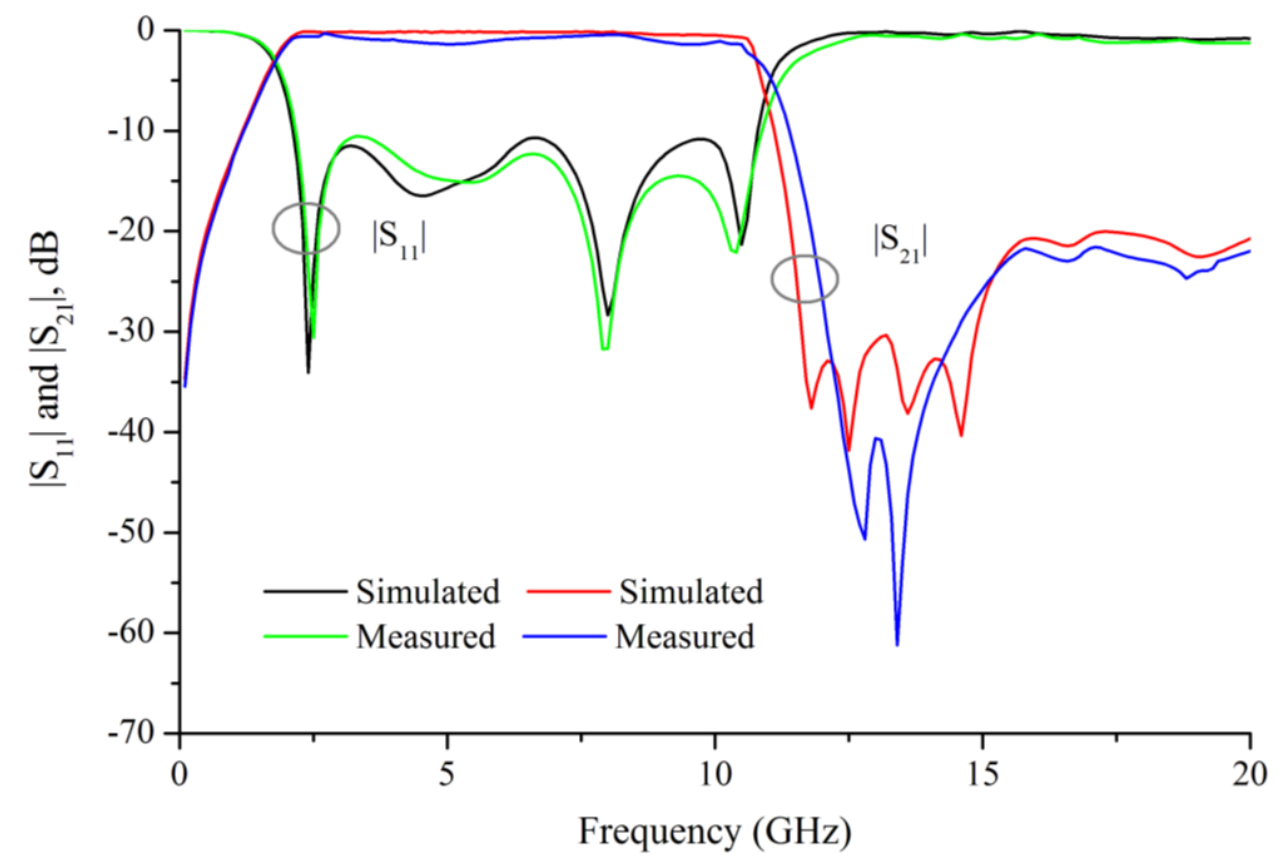

Fig. 13 Comparison of simulated and measured S-parameters

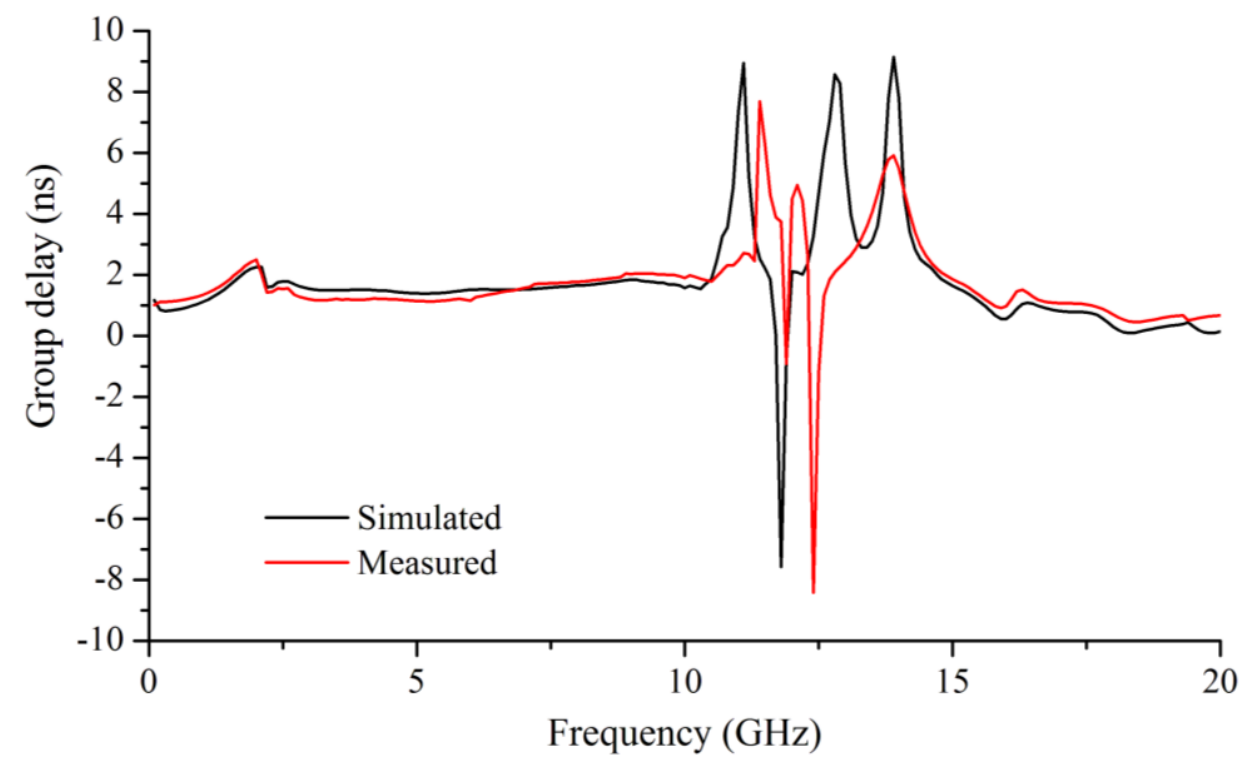

Fig. 14 Comparison of simulated and measured group delay performance 


\section{CONCLUSION}

A compact and novel ultra wideband bandpass filter based on CRLH-TL and hybrid fractal-shaped defected ground structure is designed, fabricated and measured. Effects of various design parameters have been studied to improve the performance of filter and verified experimentally. The measurement results are found in good agreement with the simulated results. The proposed UWB BPF is compact in size $\left(23 \times 20 \mathrm{~mm}^{2}\right)$ with measured return-loss and insertion-loss performance better than $10.5 \mathrm{~dB}$ and $1.1 \mathrm{~dB}$ respectively throughout the passband. Moreover, the filter is also able to achieve deep and sharpened out-of-band rejection level better than $37 \mathrm{~dB}$ with wide upper-stopband performance. Therefore the proposed geometry of UWB BPF can be a good candidate for modern small size wireless communication systems.

\section{REFERENCES}

[1] Revision of Part 15 of the commission's rules regarding ultra- wideband transmission system,"ET-Docket 98-153, First note and order, Federal Communication Commission, ” Feb 2002.

[2] Chou, T.-C., M.-H. Tsai, and C.-Y. Chen, "A low insertion loss and high selectivity UWB bandpass filter using composite right/left-handed material," Progress In Electromag. Research C, vol. 17, pp. 163-172, 2010.

[3] Huang, J.-Q. and Q.-X. Chu, "Compact UWB band-pass filter utilizing modified composite right/left-handed structure with cross coupling," Progress In Electromag. Research, vol. 107, pp. 179-186, 2010.

[4] Zhu, L., S. Sun, and W. Menzel, "Ultra-wideband (UWB) bandpass filter using multiple-mode resonator," IEEE Microw. Wireless Comp. Lett., vol. 15, no. 11, pp. 796-798, Nov 2005.

[5] Li, R. and L. Zhu, "Compact UWB bandpass filters using stub-loaded multiple-mode resonator," IEEE Microw. Wireless Comp. Lett., vol. 17, no. 1, pp. 40-42, Jan 2007.

[6] Chu, Q.-X. and X.-K. Tian, "Design of UWB bandpass filter using stepped-impedance stub-loaded resonator," IEEE Microw. Wireless Compon. Lett., vol. 20, no. 9, pp. 501-503, Sep 2010.

[7] Tang, C.-W. and M.-G. Chen, "A microstrip ultra-wideband bandpass filter with cascaded broadband bandpass and bandstop filters," IEEE Trans. Microw. Theory Tech., vol. 55, no. 11, pp. 2412- 2418, Nov 2007.

[8] Wong, S.-W. and L. Zhu, "Quadruple-mode UWB bandpass filter with improved out-of-band rejection," IEEE Microw. Wireless Compon. Lett., vol. 19, no. 3, pp. 152-154, Mar 2009.

[9] Deng, H.-W., Y.-J. Zhao, X.-S. Zhang, L. Zhang, and S.-P. Gao, "Compact quintuple-mode UWB bandpass filter with good out- of-band rejection," Progress In Electromag. Research Lett., vol. 14, pp. 111-117, 2010.

[10] Garcia-Garcia, J., J. Bonache, and F. Martin, "Application of electromagnetic bandgaps to the design of ultra-wide bandpass filters with good out-of-band performance," IEEE Trans. Microw. Theory Tech., vol. 54, no. 12, pp. 41364140, Dec 2006.

[11] Wong, S.-W. and L. Zhu, "EBG-embedded multiple-mode resonator for UWB bandpass filter with improved upperstopband performance," IEEE Microw. Wireless Compon. Lett., vol. 17, no. 6, pp. 421-423, Jun 2007.

[12] Fallahzadeh, S. and M. Tayarani, "A new microstrip UWB bandpass filter using defected microstrip structures," Journal of Electromag. Waves and Applications, vol. 24, no. 7, pp. 893-902, 2010.

[13] I-Tseng Tang, Ding-Bing Lin, Chi-Min Li, and Min-Yuan Chiu, "Ultra-wideband bandpass filter using hybrid microstrip-defected-ground structure," Microw. and Optical Tech. Lett.,vol.50, No.12, Dec 2008

[14] B. Xia1, L.-S. Wu, and J. F. Mao, “An ultra-wideband balanced bandpass filter based on defected ground structures," Progress In Electromag. Research C, vol. 25, 133-144, 2012 
[15] Lee, J.-K. and Y.-S. Kim, "Ultra-wideband bandpass filter with improved upper stopband performance using defected ground structure," IEEE Microw. Wireless Compon. Lett., vol. 20, no. 6, 316-318, Jun 2010.

[16] Jian An, Guang-Ming Wang, Wei-Dong Zeng, and Lai-Xuan Ma, "Composite right/left-handed transmission line based on koch fractal shape slot in the ground and UWB filter application", Microw. and Optical Tech. Lett., vol. 51, no. 9, Sep 2009.

[17] Xu, H.-X., Wang, G.-M., and Zhang, C.-X., 'Fractal-shaped UWB bandpass filter based on composite right/left handed transmission line," Elect. Lett., vol. 46, no.4, pp. 285-287, 2010. 